Molecules 2003, 8, 744-755

molecules

ISSN 1420-3049

http://www.mdpi.org/

\title{
Synthesis and Reactions of Some New Heterocyclic Carbohydrazides and Related Compounds as Potential Anticancer Agents
}

\author{
Abdel Kader Mansour ${ }^{*},{ }^{1}$, Mohga M. Eid ${ }^{1}$ and Nasser S. A. M. Khalil ${ }^{2}$ \\ ${ }^{1}$ Department of Chemistry, Faculty of Science, Cairo University, Giza, Egypt. \\ ${ }^{2}$ Central Laboratory for Food and Feed, Agricultural Research Centre, 9 El Gamaa St., Giza, Egypt. \\ *Author to whom correspondence should be addressed; e-mail: nasserkhalil_23@hotmail.com
}

Received: 1 June 2003; in revised form: 20 August 2003/ Accepted: 25 August 2003 / Published: 31 October 2003

\begin{abstract}
Acylation of 3-hydrazino-5,6-diphenyl-1,2,4-triazine (2) and hydrazine hydrate (7) with 4-aryl-1,3,7-triphenyl-8-oxa-1,2,6-triazaspiro[4.4]nona-2,6-dien-9-ones $\mathbf{5 a , b}$ gave the corresponding heterocyclic carbohydrazides $\mathbf{6 a , b}$ and $\mathbf{8 a , b}$ respectively. Conversion of compounds $\mathbf{8 a , b}$ into the versatile carbohydrazide derivatives 9a-g, 10, 13 and the related oxadiazoles $\mathbf{1 1}, \mathbf{1 2 a}, \mathbf{b}$ was undertaken. A primary in vitro test of compound $\mathbf{8 a}$ (concentration $10^{-4} \mathrm{M}$ ) showed activity against leukemia cell lines (CCRFCEM, K-256, MOLT-4, PRMI-8226, SR).
\end{abstract}

Keywords: Synthesis, Pyrazoles, 2-Pyrazolines, Carbohydrazides.

\section{Introduction}

Hydrazides and related compounds have been described as useful building blocks for the assembly of various heterocyclic rings [1]. A large number of aliphatic, alicyclic, aromatic and heterocyclic carbohydrazides, their derivatives and related compounds are reported to present a plethora of biological activities [2-15]. Thus, different carbohydrazides were found to be useful as medicaments especially in the treatment of inflammatory and autoimmune diseases, osteoarthritis, respiratory 
diseases, tumors, cachexia, cardiovascular diseases, fever, hemorrhage and sepsis [11]. Carbohydrazides and related compounds exhibited antifungal [2], antiviral [14], bacteriostatic [2,7,9,14], antiparasite [2,10], antituberculous [3-6], psychotropic [2], and insecticidal [15] activities. Some heterocyclic carbohydrazides are useful as antifertility agents in rats and pigeons [13]. Other carbohydrazides were reported to be components of deodorant compositions that can be used for removal of offensive odor components [16]. The 1,3,4-oxadiazoles have been reported to be biologically versatile compounds having bactericidal, fungicidal, analgesic, antiproteolytic, hypoglycemic, tranquilizing and CNS depressant properties [2]. All these facts encouraged us to synthesize some new 2-pyrazolin-3-carbohydrazides 8a,b, their derivatives 9a-g, 10, 13 and some related oxadiazoles 11, 12a,b in anticipation of expected interesting biological activities.

\section{Results and discussion}

Acylation of 3-hydrazino-5,6-diphenyl-1,2,4-triazine (2) with 4-arylidene-2-phenyl-oxazol-5(4H)ones (1a-d) has been previously reported [17] to give the corresponding N-(5,6-diphenyl-1,2,4-triazin3-yl)-carbohydrazides 3a-d (Scheme1).

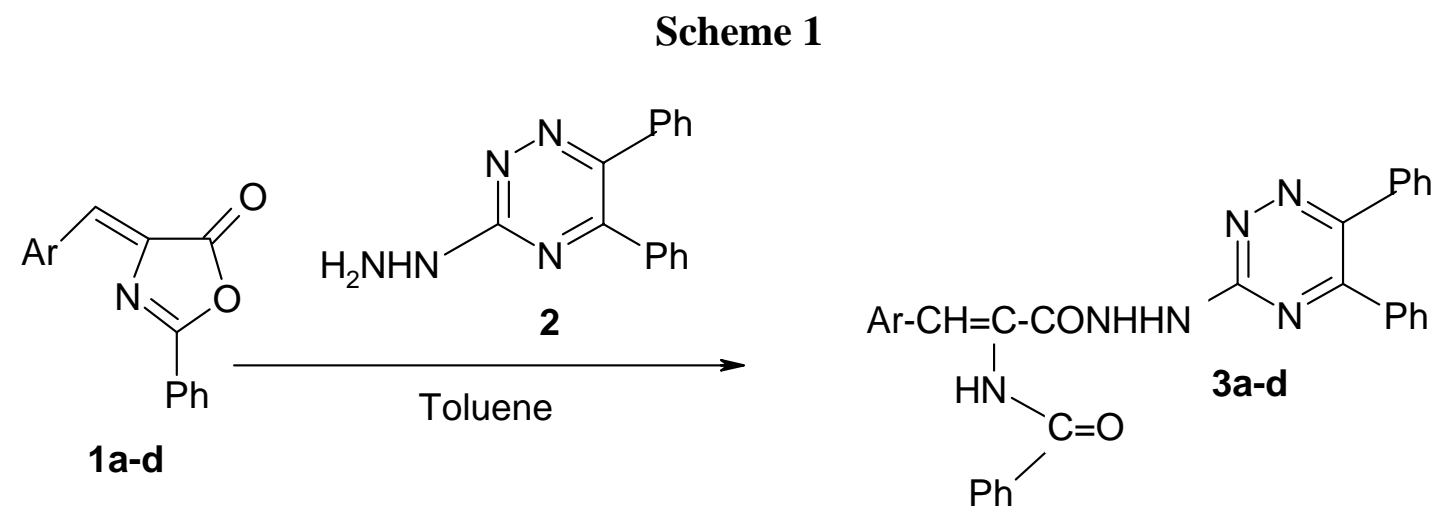

\begin{tabular}{l|l}
$\mathbf{1 , 3}$ & $\mathrm{Ar}$ \\
\hline $\mathrm{a}$ & $\mathrm{C}_{6} \mathrm{H}_{5}$ \\
b & $2-\mathrm{CH}_{3} \mathrm{OC}_{6} \mathrm{H}_{4}$ \\
c & $4-\mathrm{CH}_{3} \mathrm{OC}_{6} \mathrm{H}_{4}$ \\
d & $3,4-\left(\mathrm{CH}_{3} \mathrm{O}\right)_{2} \mathrm{C}_{6} \mathrm{H}_{3}$
\end{tabular}

We have now studied the acylation of both compound 2 and hydrazine hydrate (7) with the 4-aryl1,3,7-triphenyl-8-oxa-1,2,6-triazaspiro[4.4]nona-2,6-dien-9-ones 5a,b. Some chemical transformations leading to new functionalities have also been achieved (Scheme 2). For example, acylation of 3hydrazino-5,6-diphenyl-1,2,4-triazine (2) with the spiro compounds 5a,b [19,20] gave the corresponding novel N-(5,6-diphenyl-1,2,4-triazin-3-yl)-1,3-diphenyl-4-aryl-5-benzoylamino-2pyrazolin-5-carbohydrazides 6a,b. The structure of compounds $\mathbf{6 a}, \mathbf{b}$ was assigned based on analytical and spectral data. Thus, the IR spectra of these compounds showed bands at 3405-3396, 3277-3267 
(NH stretching), 3063-3059, 3038-3028 (aromatic CH stretching), 1709 (hydrazide $\mathrm{C}=\mathrm{O}$ stretching ), 1685-1682 (amide $\mathrm{C}=\mathrm{O}$ stretching), $1627\left(\mathrm{C}=\mathrm{N}\right.$ stretching) and 1601-1597 $\mathrm{cm}^{-1}$ (aromatic $\mathrm{C}=\mathrm{C}$ stretching).

\section{Scheme 2}

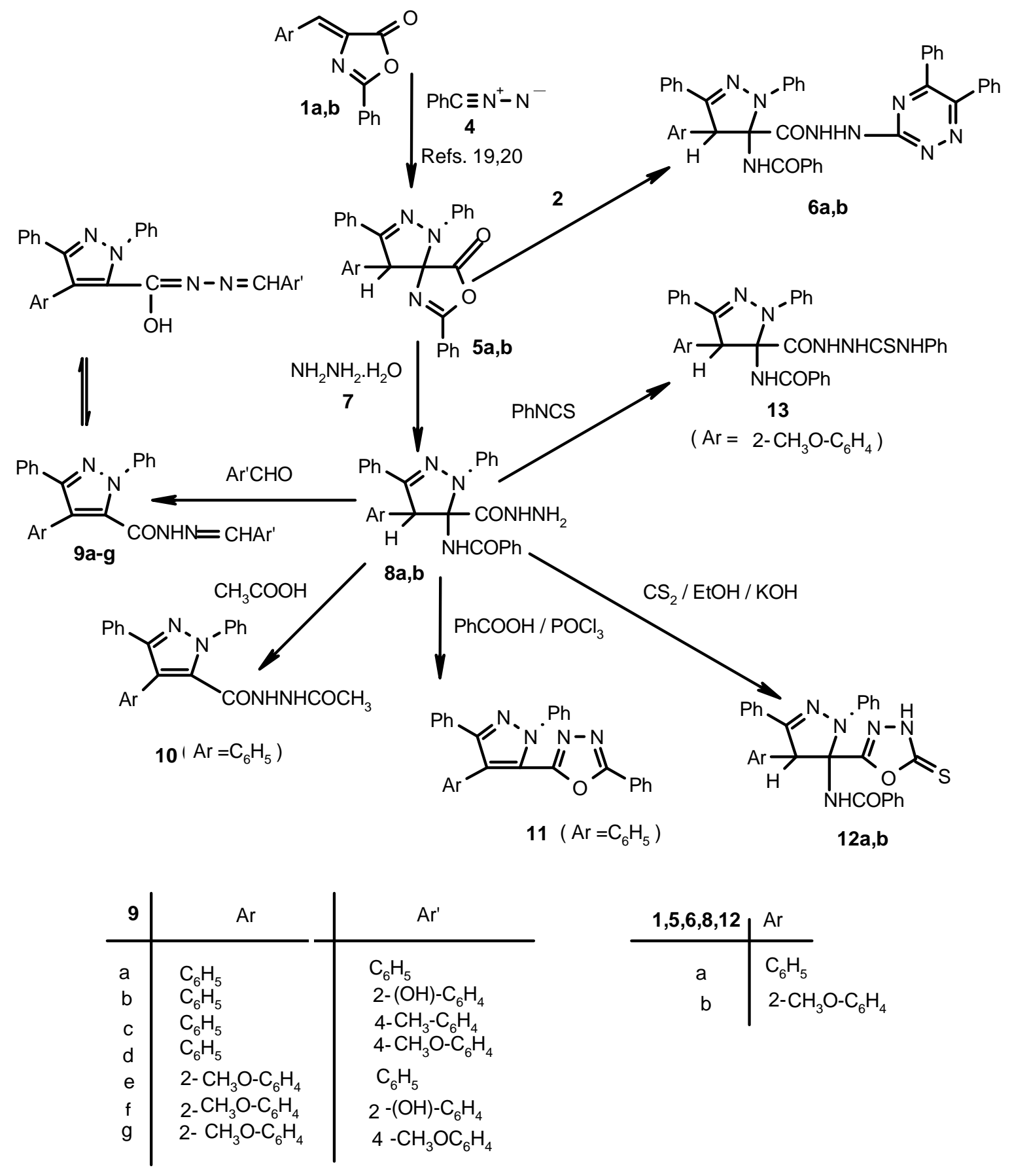


Also, the ${ }^{1} \mathrm{H}-\mathrm{NMR}$ spectra of compounds $\mathbf{6 a , b}$ revealed signals at $\delta 11.31-10.28$ (2s, hydrazide NHNH), 7.97-7.72 (s, amide NH), 7.61-6.50 (m, ArH's), 5.87-5.64 (s, pyrazolinyl CH). Moreover, the ${ }^{13} \mathrm{C}-\mathrm{NMR}$ of compounds $\mathbf{6 a}, \mathbf{b}$ showed signals consistent with their structures (cf. Experimental). Similarly, acylation of hydrazine hydrate (7) with the spiro compounds 5a,b afforded the corresponding 4-aryl-5-benzoylamino-1,3-diphenyl-2-pyrazoline-5-carbohydrazides 8a,b. The structure of compounds $\mathbf{8 a}, \mathbf{b}$ was established chemically and spectroscopically. Thus, the ${ }^{1} \mathrm{H}-\mathrm{NMR}$ spectra of these compounds not only showed the presence of the pyrazolinyl proton at $\delta$ 5.59-5.32, but also revealed the presence of $\mathrm{NH}_{2}$ protons at $\delta$ 4.61-4.54 [s, 2H (exchangeable)]. The mass spectra of compounds $\mathbf{8 a , b}$, also gave their correct parent ion peaks at m/z $475\left(\mathrm{M}^{+}, 1.3 \%\right)$, and $505\left(\mathrm{M}^{+}, 0.4 \%\right)$, respectively.

Condensation of compounds $\mathbf{8 a}, \mathbf{b}$ with aryl aldehydes and/or acetylation of compound 8a with acetic acid was found to proceed concurrently with the elimination of a benzamide molecule to give the corresponding $\mathrm{N}$-arylidene and/or N-acetoxy-4-aryl-1,3-diphenyl-pyrazole-5-carbohydrazides 9a-g and/or 10 respectively. The structures of these compounds were inferred from their analytical and spectral data. Thus, the IR spectra of compounds 9a-g showed only one carbonyl function (hydrazide $\mathrm{C}=\mathrm{O}$ ) at $1722-1670 \mathrm{~cm}^{-1}$ and that of compound $\mathbf{1 0}$ showed a carbonyl function at $1672 \mathrm{~cm}^{-1}$ (hydrazide $\mathrm{C}=\mathrm{O}$ overlapped with acetyl $\mathrm{C}=\mathrm{O}$ ). The ${ }^{1} \mathrm{H}-\mathrm{NMR}$ spectra of $\mathbf{9 a - g}$ and $\mathbf{1 0}$ not only showed the absence of both the pyrazolinyl proton at $\delta$ 5.59-5.32 and the $\mathrm{NH}_{2}$ protons at $\delta 4.61-4.54$, but also the presence of the $\mathrm{N}=\mathrm{CH}$ in compounds $9 \mathbf{a - g}(\delta 8.30-7.65)$ and the acetyl protons of compound $\mathbf{1 0}(\delta 1.86)$. The IR spectra of compounds 9a-g as well as their ${ }^{1} \mathrm{H}-\mathrm{NMR}$ spectra (relative proton integration ratio) showed the presence of both hydrazide (25-63.3\%) and hydrazole (36.7-75\%) tautomeric structures. The mass spectra of compounds 9a,c gave their correct parent ion peaks at m/z $442\left(\mathrm{M}^{+}, 13.7 \%\right)$ and $456\left(\mathrm{M}^{+}\right.$, $12 \%)$, respectively.

Treatment of compound 8a with benzoic acid and phosphorous oxychloride was found to proceed via concurrent cyclocondensation and elimination of a benzamide molecule to give the 5-phenyl-2(1,3,4-triphenylpyrazol-5-yl)-1,3,4-oxadiazole (11). The mass spectrum of compound 11 showed its correct parent ion peak at $\mathrm{m} / \mathrm{z} 440\left(\mathrm{M}^{+}, 49.9 \%\right)$.

Compounds 8a,b reacted with carbon disulfide in ethanolic $\mathrm{KOH}$ to yield the corresponding 2-(4aryl-1,3-diphenyl-5-benzoylamino-2-pyrazolin-5-yl)-1,3,4-oxadiazole-5(4H)-thiones (12a,b). The absence of the characteristic weak S-H stretching bands at 2600-2550 and the appearance of the strong $\mathrm{C}=\mathrm{S}$ stretching bands at 1069-1064 $\mathrm{cm}^{-1}$ in the IR spectra of compounds 12a,b confirmed their thione form and excluded the tautomeric thiol structure. The IR spectra of compounds 12a,b showed also bands at $1199-1019 \mathrm{~cm}^{-1}$ (=C-O-C= stretching). The ${ }^{1} \mathrm{H}-\mathrm{NMR}$ spectra revealed the presence of the NH oxadiazolyl proton at $\delta 14.60-14.52$ and the $\mathrm{CH}$ pyrazolinyl proton at $\delta$ 6.32-5.90.

Reaction of compound $\mathbf{8 b}$ with phenyl isothiocyanate gave N-(N-phenylthiocarbamoyl)-5benzoylamino-1,3-diphenyl-4-(2-methoxyphenyl)-2-pyrazolin-5-carbohydrazide (13). Elemental and spectral data are consistent with the structure assigned to this compound (cf. Experimental). 


\section{Biological Evaluation}

A primary in vitro test of compound $8 \mathbf{a}$ (concentration $10^{-4} \mathrm{M}$ ) showed activity against Leukemia cell lines (CCRF-CEM, K-562, MOLT-4, PRMI-8226, SR). Further investigation is in progress.

\section{Experimental}

\section{General}

All melting points are uncorrected. IR spectra (KBr disks) were recorded on a Perkin-Elmer 1430 spectrometer. The abbreviations str. and bend. are used to indicate stretching and bending bands, respectively . ${ }^{1} \mathrm{H}-\mathrm{NMR}$ and ${ }^{13} \mathrm{C}$-NMR were measured with a Varian GEMINI 200 spectrometer (200 $\mathrm{MHz}$ for ${ }^{1} \mathrm{H}-\mathrm{NMR}$; $50 \mathrm{MHz}$ for ${ }^{13} \mathrm{C}-\mathrm{NMR}$ ). The abbreviation exch. is used to indicate exchangeable protons. Mass spectra were recorded on a GCMS - QP 1000 EX (70EV) spectrometer. Elemental analyses were carried out at the Microanalytical Center, Cairo University. Anticancer screening of compound 8a was carried out at the National Cancer Institute - National Institutes of Health, Bethesda, Maryland, United States of America. The starting materials 3-hydrazino-5,6-diphenyl-1,2,4-triazine (2) [18] and 4-aryl-1,3,7-triphenyl-8-oxa-1,2,6-triazaspiro-[4.4]nona-2,6-dien-9-ones 5a,b [19,20] were prepared as reported.

General procedure for the preparation of N-(5,6-diphenyl-1,2,4-triazin-3-yl)-4-aryl-5-benzoylamino1,3-diphenyl-2-pyrazoline-5-carbohydrazides $(\mathbf{6 a , b})$.

Each of compounds $\mathbf{5 a , b}(1 \mathrm{mmol})$ was added to a solution of compound $\mathbf{2}(1 \mathrm{mmol})$ in toluene (10 $\mathrm{mL}$ ). The reaction mixture was then heated under reflux for $1 \frac{1}{2}$ hour, concentrated, and diluted with petroleum ether (bp. $\left.40-60^{\circ} \mathrm{C}\right)$. After decantation, the residue obtained was boiled in ethanol $(5 \mathrm{~mL})$, filtered, and cooled. The formed precipitate was collected by filtration and recrystallized from ethanol giving colorless crystals of $\mathbf{6 a}, \mathbf{b}$.

N-(5,6-Diphenyl-1,2,4-triazin-3-yl)-5-benzoylamino-1,3,4-triphenyl-2-pyrazoline-5-carbohydrazide

(6a). Using the general procedure, 5a gave 6a (73\%); mp. $218-219^{\circ} \mathrm{C}$; IR (cm $\left.{ }^{-1}\right): 3405,3277$ (NH str.), 3063, 3038 (aromatic CH str.), 2922, 2866, 2855 (pyrazolinyl aliphatic $\mathrm{CH}$ str.), 1709 (hydrazide $\mathrm{C}=\mathrm{O}$ str.), 1685 (amide $\mathrm{C}=\mathrm{O}$ str.), 1627 ( $\mathrm{C}=\mathrm{N}$ str.), 1601 (aromatic $\mathrm{C}=\mathrm{C}$ str.), 1505, 1480 ( $\mathrm{NH}$ bend.), 1365, 1312, 1283, 1251 (C-N str.), 762, 697 (aromatic CH bend.); ${ }^{1} \mathrm{H}-\mathrm{NMR}$ (DMSO-d 6 ) $\delta 11.31,10.31$ [2s, 2H (exch.), hydrazide NHNH], 7.72 [s, 1H (exch.), amide NH], 7.57-6.82 (m, 30H, ArH's), 5.64 (s, $1 \mathrm{H}$, pyrazolinyl $\mathrm{CH}) ;{ }^{13} \mathrm{C}-\mathrm{NMR}\left(\mathrm{DMSO}_{\mathrm{d}}\right) \delta 171.5,164.8(\mathrm{C}=\mathrm{O}), 157,152,146.5,142.5(\mathrm{C}=\mathrm{N})$, 136.2, 134.6, 132.2, 131.5 (aromatic C), 130.5, 129.5, 129.4, 128.7, 128.6, 128.5, 128.2, 127.6, 127.3, 127.2, 127.0. 126.2 (aromatic CH), 81.6 (pyrazolinyl C), 63.5 (pyrazolinyl CH); Anal. for $\mathrm{C}_{44} \mathrm{H}_{34} \mathrm{~N}_{8} \mathrm{O}_{2}$ Calcd.: C, 74.77; H, 4.85; N, 15.85. Found: C, 74.60; H, 5.00, N, 15.70. 
N-(5,6-Diphenyl-1,2,4-triazin-3-yl)-5-benzoylamino-1,3-diphenyl-4-(2-methoxyphenyl)-2-pyrazoline5-carbohydrazide (6b). Using the general procedure, 5b gave $6 \mathbf{b}(41 \%) ; \mathrm{mp} .210-212^{\circ} \mathrm{C}$; IR $\left(\mathrm{cm}^{-1}\right)$ : 3396, 3267 (NH str.), 3059, 3028 (aromatic CH str.), 2964, 2932, 2883, $2833\left(\mathrm{OCH}_{3}\right.$ and pyrazolinyl aliphatic $\mathrm{CH}$ str.), 3059, 3028, 2997 (aromatic $\mathrm{CH}$ str.), 1709 (hydrazide $\mathrm{C}=\mathrm{O}$ str.), 1682 (amide $\mathrm{C}=\mathrm{O}$ str.), 1597(aromatic C=C str.), 1495, 1473, 1445 (NH bend.), 1358, 1331, 1290, 1252 (C-N str.), 1067, 1028 (C-O str. of $\mathrm{OCH}_{3}$ ), 762, 692 (aromatic CH bend.); ${ }^{1} \mathrm{H}-\mathrm{NMR}\left(\mathrm{DMSO}-\mathrm{d}_{6}\right) \delta 11.23,10.28$ [2 brs, 2H (exch.), hydrazide NHNH], 7.97 [s, 1H (exch.), amide NH] 7.61-6.50 (m, 29H, ArH's), 5.87 (s, 1H, pyrazolinyl $\mathrm{CH}), 3.60\left(\mathrm{~s}, 3 \mathrm{H}, \mathrm{OCH}_{3}\right) ;{ }^{13} \mathrm{C}-\mathrm{NMR}\left(\mathrm{DMSO}_{6}\right) \delta$ 171.64, 164.30, $(\mathrm{C}=\mathrm{O}), 157.28,146.84$, 142.78, $136.41(\mathrm{C}=\mathrm{N}), 135.95,134.76,132.74,132.49,132.45,132.41,131.46$ (aromatic C), 130.54, 129.70. 129.31, 129.20, 128.74, 128.64, 128.40, 128.29, 126.86, 126.08, 125.89, 121.68, 120.33, 115.15, 115.09, 114.99 (aromatic CH), 110.04 (pyrazolinyl C), 80.55 (pyrazolinyl $\mathrm{CH}$ ), $55.04\left(\mathrm{OCH}_{3}\right)$; Anal. for $\mathrm{C}_{45} \mathrm{H}_{36} \mathrm{~N}_{8} \mathrm{O}_{3}$ Calcd.: C, 73.35; H, 4.92; N, 15.21. Found: C, 73.50; H, 5.00; N, 15.40 .

General procedure for the preparation of 4-aryl-5-benzoylamino-1,3-diphenyl-2-pyrazoline-5carbohydrazides (8a,b). To a suspension of each of compounds $\mathbf{5 a}, \mathbf{b}(1 \mathrm{mmol})$ in methanol $(20 \mathrm{~mL})$ was added $80 \%$ hydrazine hydrate $(0.5 \mathrm{~mL})$. While the reaction mixture of compound 8a was shaken for ten minutes at room temperature, that of compound $\mathbf{8 b}$ was heated under reflux for one hour. The reaction mixtures of each compound were then left overnight at room temperature. The colorless precipitate formed was collected by filtration and recrystallized from methanol to give colorless crystals of $\mathbf{8 a , b}$.

5-Benzoylamino-1,3,4-triphenyl-2-pyrazoline-5-carbohydrazide (8a). Using the general procedure, 5a gave 8a (73\%); mp. 214-215 ${ }^{\circ} \mathrm{C}$; IR ( $\left.\mathrm{cm}^{-1}\right)$ : 3400, 3325 (NH, $\mathrm{NH}_{2}$ str.), 3065, 3034 (aromatic $\mathrm{CH}$ str.), 2930 (pyrazolinyl $\mathrm{CH}$ str.), 1669 ( $\mathrm{C}=\mathrm{O}$ str.), 1625 (C=N str.), 1601 (aromatic $\mathrm{C}=\mathrm{C}$ str.), 1503, 1480 (NH bend.), 1374, 1321, 1280, 1243 (C-N str.), 750, 696 (aromatic CH bend.); ${ }^{1} \mathrm{H}-\mathrm{NMR}$ (DMSO-d $\left.{ }_{6}\right) \delta$ 10.05 [s, 1H (exch.), hydrazide NH], 8.07 [s, 1H (exch.), amide NH], 7.60-6.86 (m, 20H, ArH's), 5.32 (s, 1H, pyrazolinyl $\mathrm{CH}$ ), 4.54[s, 2H (exch.), $\left.\mathrm{NH}_{2}\right]$; MS m/z $475\left(\mathrm{M}^{+}, 1.3 \%\right)$; Anal. for $\mathrm{C}_{29} \mathrm{H}_{25} \mathrm{~N}_{5} \mathrm{O}_{2}$ Calcd.: C, 73.24; H, 5.30; N, 14.73. Found: C, 73.20; H, 5.25; N, 14.60.

5-Benzoylamino-1,3-diphenyl-4-(2-methoxyphenyl)-2-pyrazoline-5-carbohydrazide (8b). Using the general procedure, $\mathbf{5 b}$ gave $\mathbf{8 b}(74 \%)$; mp. $230^{\circ} \mathrm{C}$; IR $\left(\mathrm{cm}^{-1}\right)$ : 3384, 3334,3284 (NH, $\mathrm{NH}_{2}$ str.), 3061, 3025 (aromatic $\mathrm{CH}$ str.), 2954, 2942, 2901, $2835\left(\mathrm{OCH}_{3}\right.$ and pyrazolinyl aliphatic $\mathrm{CH}$ str.), 1684 (hydrazide $\mathrm{C}=\mathrm{O}$ str.), 1672 (amide $\mathrm{C}=\mathrm{O}$ str.), 1636 ( $\mathrm{C}=\mathrm{N}$ str.), 1604 (aromatic $\mathrm{C}=\mathrm{C}$ str.), 1497, 1475 (NH bend.), 1375, 1294, 1250 (C-N str.), 1167, 1028 (C-O str. of $\mathrm{OCH}_{3}$ ), 750, 692 (aromatic $\mathrm{CH}$ bend.); ${ }^{1} \mathrm{H}-\mathrm{NMR}\left(\mathrm{DMSO}_{-} \mathrm{d}_{6}\right) \delta 10.25$ [s, $1 \mathrm{H}$ (exch.), hydrazide NH], 8.18 [s, 1H (exch.), amide NH], 7.56-6.49 (m, 19H, ArH's), 5.59 (s, 1H, pyrazolinyl CH), 4.61[s, 2H (exch.), $\left.\mathrm{NH}_{2}\right], 3.82$ (s, 3H, $\left.\mathrm{OCH}_{3}\right) ; \mathrm{MS} \mathrm{m} / \mathrm{z} 505\left(\mathrm{M}^{+}, 0.4 \%\right)$; Anal. for $\mathrm{C}_{30} \mathrm{H}_{27} \mathrm{~N}_{5} \mathrm{O}_{3}$ Calcd.: C, 71.27; H, 5.38; N, 13.85. Found: C, $71.30 ; \mathrm{H}, 5.50 ; \mathrm{N}, 14.00$. 
N-Arylidene-4-aryl-1,3-diphenylpyrazole-5-carbohydrazides (9a-g).

General Procedure A: To a solution of each of compounds 8a,b (1 mmol) in either acetic acid (12 mL) or n-butanol $(20 \mathrm{~mL})$ was added the appropriate aryl aldehyde $(1 \mathrm{mmol})$ and the reaction mixture was then heated under reflux for a time as shown as in Table 1. The crystalline colorless precipitates of compounds 9a-d,f were collected by filtration, washed with methanol, and recrystallized from the appropriate solvent. Pure 9e,g were obtained from their reaction mixtures via dilution with water, collection of the formed precipitate by filtration, and recrystallization from the appropriate solvent.

\section{Table 1}

\begin{tabular}{|l|l|l|l|}
\hline 9 & Reaction Solvent & Reflux Time & Recrystallization Solvent \\
\hline a & n-Butanol & 3 hours & EtOH \\
b & Acetic acid & 20 minutes & n-Butanol \\
c & Acetic acid & $1 \frac{1}{2}$ hour & Acetic acid \\
d & Acetic acid & $1 \frac{1}{2}$ hour & Acetic acid \\
e & Acetic acid & 2 hours & Ethanol \\
f & Acetic acid & $1 \frac{1}{4}$ hour & Ethanol \\
g & Acetic acid & 2 hours & Ethanol \\
\hline
\end{tabular}

General Procedure B: To compound 8a $(1 \mathrm{mmol})$ was added 4-methylbenzaldehyde (1 mmol) and 3 drops of piperidene. The reaction mixture was then heated (oil bath) at $120^{\circ} \mathrm{C}$ till complete dissolution which was followed by an immediate resolidification. The reaction mixture was allowed to cool to room temperature, diluted with methanol, and the formed colorless crystalline product was collected by filtration and recrystallized from ethanol giving colorless crystals of 9c, identical with the product 9c obtained from general procedure A (mp. and mixed mp. as well as analytical and spectral data).

N-Benzylidene-1,3,4-triphenylpyrazole-5-carbohydrazide (9a). Using general procedure A, 8a gave 9a (34\%); mp. 229-230 ${ }^{\circ} \mathrm{C}$; IR ( $\left.\mathrm{cm}^{-1}\right)$ : 3584-3291 (OH str. of hydrazole tautomer), 3213 (NH str.), 3066, 3028 (aromatic $\mathrm{CH}$ str.), 1674 (hydrazide $\mathrm{C}=\mathrm{O}$ str.) 1655 ( $\mathrm{C}=\mathrm{N}$ str.), 1597 (aromatic $\mathrm{C}=\mathrm{C}$ str.), 1556, 1500 (NH bend.), 1365, 1313, 1275, 1246 (C-N str.), 760, 692 (aromatic CH bend.); ${ }^{1} \mathrm{H}-\mathrm{NMR}\left(\mathrm{CDCl}_{3}\right)$ $\delta$ 10.03, 8.43 [ 2brs, $1 \mathrm{H}$ (exch.), hydrazide (53.7\%) - hydrazole (46.3\%) tautomeric proton], 7.73-7.07 $(\mathrm{m}, 21 \mathrm{H}, \mathrm{ArH}$ 's, $\mathrm{N}=\mathrm{CH}) ; \mathrm{Ms} \mathrm{m} / \mathrm{z} 442\left(\mathrm{M}^{+}, 13.7 \%\right)$; Anal. for $\mathrm{C}_{29} \mathrm{H}_{22} \mathrm{~N}_{4} \mathrm{O}$ Calcd.: $\mathrm{C}, 78.71 ; \mathrm{H}, 5.01 ; \mathrm{N}$, 12.66. Found: C, 78.80; H, 5.20; N, 12.80 .

N-(2-Hydroxybenzylidene)-1,3,4-triphenylpyrazole-5-carbohydrazide (9b). Using general procedure A, 8a gave 9b (88\%); mp.283-285 ${ }^{\circ}$; IR ( $\left.\mathrm{cm}^{-1}\right)$ : 3200-2500 (OH str.), 3178 (NH str.), 3055, 3007 (aromatic $\mathrm{CH}$ str.), 1722 (hydrazide $\mathrm{C}=\mathrm{O}$ str.), 1651(C=N str.), 1601 (aromatic $\mathrm{C}=\mathrm{C}$ str.), 1545, 1499 (NH bend.), 1367, 1331, 1313, 1292, 1277, 1250, 1186, 1184, 1149 [C-N, C-O (phenolic) str.], 758, 
692 (aromatic CH bend.); ${ }^{1} \mathrm{H}-\mathrm{NMR}\left(\mathrm{DMSO}_{-} \mathrm{d}_{6}\right) \delta 12.36$ [s, $1 \mathrm{H}$ (exch.), 2-(O프)- $\left.\mathrm{C}_{6} \mathrm{H}_{4}\right]$ 10.70, $9.96[2 \mathrm{~s}$, $1 \mathrm{H}$ (exch.), hydrazide (63.3\%) - hydrazole (36.7\%) tautomeric proton], $8.30(\mathrm{~s}, 1 \mathrm{H}, \mathrm{N}=\mathrm{CH}), 7.72-6.73$ (m, $19 \mathrm{H}$, ArH's); Anal. for $\mathrm{C}_{29} \mathrm{H}_{22} \mathrm{~N}_{4} \mathrm{O}_{2}$ Calcd.: C, 75.97; H, 4.83; N, 12.22. Found: C, 76.00; H, 4.76; $\mathrm{N}, 12.00$.

$N$-(4-Methylbenzylidene)-1,3,4-triphenylpyrazole-5-carbohydrazide (9c). Using the general procedures A and/or B, 8a gave 9c (67\%) and / or (70\%), respectively; mp. 222-223 ${ }^{\circ} \mathrm{C}$; IR $\left(\mathrm{cm}^{-1}\right)$ : 3342-2400 (OH str. of hydrazole tautomer), 3197 (NH str.), 3052, 3000 (aromatic $\mathrm{CH}$ str.), 2947, 2919 (CH str. of $\mathrm{CH}_{3}$ ), 1672 (hydrazide $\mathrm{C}=\mathrm{O}$ str.) 1653 ( $\mathrm{C}=\mathrm{N}$ str.), 1603 (aromatic $\mathrm{C}=\mathrm{C}$ str.), 1564, 1500 ( $\mathrm{NH}$ bend.), 1372, 1335, 1322, 1311, 1292, 1279, 1256, 1211-1027 [C-O (of hydrazole tautomer), C-N str.], 765,757, 702, 684 (aromatic CH bend.); ${ }^{1} \mathrm{H}-\mathrm{NMR}\left(\mathrm{CDCl}_{3}\right) \delta 10.45,8.50$ [2s, $1 \mathrm{H}$ (exch.), hydrazide (61.0\%) - hydrazole (39.0\%) tautomeric proton], 7.65-7.08 (m, 20H, ArH's, $\mathrm{N}=\mathrm{CH}), 2.33\left(\mathrm{~s}, 3 \mathrm{H}, \mathrm{CH}_{3}\right)$; MS m/z $456\left(\mathrm{M}^{+}, 12.0 \%\right)$; Anal. for $\mathrm{C}_{30} \mathrm{H}_{24} \mathrm{~N}_{4} \mathrm{O}$ Calcd.: $\mathrm{C}, 78.92 ; \mathrm{H}, 5.30 ; \mathrm{N}, 12.27$. Found: $\mathrm{C}, 78.90$; $\mathrm{H}, 5.40 ; \mathrm{N}, 12.40$.

$N$-(4-Methoxybenzylidene)-1,3,4-triphenylpyrazole-5-carbohydrazide (9d). Using general procedure A, 8a gave 9d (83\%); mp. 245-247 ${ }^{\circ} \mathrm{C}$; IR $\left(\mathrm{cm}^{-1}\right)$ : 3713-2555 (OH str.of hydrazole tautomer), $3194(\mathrm{NH}$ str.), 3055, 3006 (aromatic $\mathrm{CH}$ str.), 2967, 2927, 2838 ( $\mathrm{CH}$ str. of $\mathrm{OCH}_{3}$ ), 1673 (hydrazide $\mathrm{C}=\mathrm{O}$ str.), 1651 ( $\mathrm{C}=\mathrm{N}$ str.), 1604 (aromatic $\mathrm{C}=\mathrm{C}$ str.), 1565, 1506 ( $\mathrm{NH}$ bend.), 1369, 1306, 1251, 1172-1028 [C$\mathrm{O}, \mathrm{C}-\mathrm{N}$ str.), 762,701 (aromatic $\mathrm{CH}$ bend.); ${ }^{1} \mathrm{H}-\mathrm{NMR}\left(\mathrm{CDCl}_{3}\right) \delta 9.93,8.40[2 \mathrm{~s}, 1 \mathrm{H}$ (exch.), hydrazide $(52.2 \%)$ - hydrazole (47.8\%) tautomeric proton], 7.69-6.83 (m, 20H, ArH's, N=CH), $3.84(\mathrm{~s}, 3 \mathrm{H}$, $\mathrm{OCH}_{3}$ ); Anal. for $\mathrm{C}_{30} \mathrm{H}_{24} \mathrm{~N}_{4} \mathrm{O}_{2}$ Calcd.: C, 76.25; H, 5.12; N, 11.86. Found: C, 76.40; H, 5.08; N, 12.00 .

N-Benzylidene-1,3-diphenyl-4-(2-methoxyphenyl)-pyrazole-5-carbohydrazide (9e). Using general procedure A, 8b gave 9e (80\%); mp. $175-176^{\circ} \mathrm{C}$; IR $\left(\mathrm{cm}^{-1}\right): 3200-2500(\mathrm{OH}$ str. of hydrazole tautomer), 3196 (NH str.), 3067 (aromatic $\mathrm{CH}$ str.), 2987, 2930, $2856\left(\mathrm{CH}\right.$ str. of $\left.\mathrm{OCH}_{3}\right), 1670$ (hydrazide $\mathrm{C}=\mathrm{O}$ str.) 1651 ( $\mathrm{C}=\mathrm{N}$ str.), 1612, 1595 (aromatic $\mathrm{C}=\mathrm{C}$ str.), 1556, 1499 ( $\mathrm{NH}$ bend.), 1364, 1273,1242-1074 (C-O, C-N str.), 760, 700, 692 (aromatic CH bend.); ${ }^{1} \mathrm{H}-\mathrm{NMR}\left(\mathrm{CDCl}_{3}\right) \delta 9.83,9.43$ [2s, $1 \mathrm{H}$ (exch.), hydrazide (27.8\%) - hydrazole (72.2\%) tautomeric proton], 7.72-6.95 (m, 20H, ArH's, $\mathrm{N}=\mathrm{CH}), 3.85\left(\mathrm{~s}, 3 \mathrm{H}, \mathrm{OCH}_{3}\right)$; Anal. for $\mathrm{C}_{30} \mathrm{H}_{24} \mathrm{~N}_{4} \mathrm{O}_{2}$ Calcd.: C, 76.25; H, 5.12; N, 11.86. Found: $\mathrm{C}$, 76.40; H, 5.10; N, 11.60 .

$N$-(2-Hydroxybenzylidene)-1,3-diphenyl-4-(2-methoxyphenyl)-pyrazole-5-carbohydrazide (9f). Using general procedure A, 8b gave 9f $(81 \%)$; mp. $207-208^{\circ} \mathrm{C} ;{ }^{1} \mathrm{H}-\mathrm{NMR}\left(\mathrm{CDCl}_{3}\right): \delta 10.69[\mathrm{~s}, 1 \mathrm{H}$ (exch.), $\mathrm{OH}$ ], 9.37 [s, $1 \mathrm{H}$ (exch.), NH], 7.89 (s, 1H, N=CH), 7.67-6.84 (m, 18H, ArH's), 3.84 (s, 3H, $\mathrm{OCH}_{3}$ ); Anal. for $\mathrm{C}_{30} \mathrm{H}_{24} \mathrm{~N}_{4} \mathrm{O}_{3}$ Calcd.: C, 73.75; H, 4.95; N, 11.47. Found: C, 73.80; H, 5.00; N, 11.62.

$\mathrm{N}$-(4-Methoxybenzylidene)-1,3-diphenyl-4-(2-methoxyphenyl)-pyrazole-5-carbohydrazide (9g). Using general procedure A, $\mathbf{8 b}$ gave $\mathbf{9 g}(85 \%)$; mp. $178-180^{\circ} \mathrm{C}$; IR $\left(\mathrm{cm}^{-1}\right)$ : 3645-3266 (OH str. of hydrazole 
tautomer), 3175 (NH str.), 3068, 3005 (aromatic $\mathrm{CH}$ str.), 2962, 2933, 2902, 2837(CH str. of $\mathrm{OCH}_{3}$ ), 1685 (hydrazide $\mathrm{C}=\mathrm{O}$ str.) $1650(\mathrm{C}=\mathrm{N}$ str.), 1603 (aromatic $\mathrm{C}=\mathrm{C}$ str.), 1547, 1509 ( $\mathrm{NH}$ bend.), 1366, 1307,1254 , 1170-1026 (C-O, C-N str.) 758, 694 (aromatic $\mathrm{CH}$ bend.); ${ }^{1} \mathrm{H}-\mathrm{NMR}\left(\mathrm{CDCl}_{3}\right): \delta 9.74,9.34$ [2s, $1 \mathrm{H}$ (exch.), hydrazide (25.0\%) - hydrazole (75.0\%) tautomeric proton], 7.72-6.86 (m, 19H, ArH's, $\mathrm{N}=\mathrm{CH}), 3.83\left(\mathrm{~s}, 6 \mathrm{H}, \mathrm{OCH}_{3}\right)$; Anal. for $\mathrm{C}_{31} \mathrm{H}_{26} \mathrm{~N}_{4} \mathrm{O}_{3}$ Calcd.: C, 74.08; H, 5.21; N, 11.15. Found: $\mathrm{C}$, $74.12 ; \mathrm{H}, 5.10 ; \mathrm{N}, 11.60$.

$\mathrm{N}$-Acetyl-1,3,4-triphenylpyrazole-5-carbohydrazide (10). To compound $\mathbf{8 a}$ (1 mmol) was added acetic acid $(15 \mathrm{~mL})$ and the reaction mixture was heated under reflux for $1 \frac{1}{4}$ hour. After cooling, the reaction mixture was poured onto water and the formed precipitate was collected by filtration and recrystallized from ethanol as colorless crystals of $\mathbf{1 0}(83 \%)$; mp. $237-238^{\circ} \mathrm{C}$; IR ( $\left.\mathrm{cm}^{-1}\right)$ : 3194 (NH str.), 3055, 3009 (aromatic $\mathrm{CH}$ str.), 2968, 2914, 2839 ( $\mathrm{CH}$ str. of $\left.\mathrm{CH}_{3}\right), 1672(\mathrm{C}=\mathrm{O}$ str.), 1651 (C=N str.), 1605 (aromatic $\mathrm{C}=\mathrm{C}$ str.), 1564, 1512, 1500 (NH bend.), 1371, 1306, 1279, 1250 (C-N str.), 772, 768, 704, 690 (aromatic $\mathrm{CH}$ bend.); ${ }^{1} \mathrm{H}-\mathrm{NMR}\left(\mathrm{DMSO}_{6}\right): \delta$ 10.76, 9.98 [2s, $2 \mathrm{H}$ (exch.), hydrazide NHNH], 7.97-7.26 (m, 15H, ArH's), 1.85 (s, 3H, $\underline{\mathrm{H}}_{3} \mathrm{CO}$ ); Anal. for $\mathrm{C}_{24} \mathrm{H}_{20} \mathrm{~N}_{4} \mathrm{O}_{2}$ Calcd.: C, 72.71; H, 5.08; $\mathrm{N}$, 14.13. Found: C, 72.80; H, 5.10; N, 14.50.

2-(1,3,4-Triphenylpyrazol-5-yl)-5-phenyl-1,3,4-oxadiazole (11). A mixture of compound $8 \mathbf{a}$ (1 mmol), benzoic acid $(1 \mathrm{mmol})$, and phosphororus oxychloride $(1 \mathrm{mmol})$ was heated under reflux for $1 \frac{1}{2}$ hour. After cooling to room temperature, the reaction mixture was poured onto crushed ice, stirred, decanted and diluted with water. The residue obtained after a second decantation was boiled with methanol. The solidified residue obtained on cooling was collected by filtration and recrystallized from N,Ndimethylformamide as colorless crystals of $11(23 \%)$; mp. $185^{\circ} \mathrm{C} ;{ }^{1} \mathrm{H}-\mathrm{NMR}\left(\mathrm{DMSO}-\mathrm{d}_{6}\right): \delta$ 7.69-7.34 (m, ArH's); MS m/z $440\left(\mathrm{M}^{+}\right.$, 49.9); Anal. for $\mathrm{C}_{29} \mathrm{H}_{20} \mathrm{~N}_{4} \mathrm{O}$ Calcd.: C, 79.07; H, 4.57; N, 12.72. Found: C, 79.10; H, 4.62; N, 12.69 .

General procedure for the preparation of 2-(4-Aryl-5-benzoylamino-1,3-diphenyl-2-pyrazolin-5-yl)1,3,4-oxadiazole-5(4H)-thiones (12a,b). To a solution containing 95\% ethanol $(5 \mathrm{~mL})$ and potassium hydroxide ( $1 \mathrm{mmol}$, dissolved in the least amount of water), were added compounds $\mathbf{8 a}$,b $(1 \mathrm{mmol})$ followed by carbon disulfide $(1.5 \mathrm{mmol})$. The reaction mixture was heated under reflux for 3 hours till all the evolution of hydrogen sulfide ceased. After decantation, the supernatant solution was evaporated, diluted with water (while a milky solution was obtained), and acidified with hydrochloric acid containing ice. The reaction mixture was allowed to stand at room temperature for 15 minutes, filtered, and the solid obtained was washed well with water and dried at room temperature. The crude products were then recrystallized from ethanol as colorless crystals of $\mathbf{1 2 a}, \mathbf{b}$.

2-(5-Benzoylamino-1,3,4-triphenyl-2-pyrazolin-5-yl)-1,3,4-oxadiazole-5(4H)-thione (12a). Using the general procedure, 8a gave 12a (81\%); mp. 236-237 $\mathrm{C}$; IR ( $\left.\mathrm{cm}^{-1}\right): 3421,3157$ (NH str.), 3063, 3030 (aromatic CH str.), 2945, 2926, 2852 (pyrazolinyl CH str.), 1683 (amide $\mathrm{C}=\mathrm{O}$ str.), 1600 (aromatic 
$\mathrm{C}=\mathrm{C}$ str.), 1507, 1489, 1469 (NH bend.), 1331, 1286, 1255 (C-N str.), 1199, 1153,1019 (=C-O-C= str.), 1064 (C=S str.), 761, 649 (aromatic $\mathrm{CH}$ bend.); ${ }^{1} \mathrm{H}-\mathrm{NMR}$ (DMSO-d 6 ): $\delta 14.60$ [brs, $1 \mathrm{H}$ (exch.), oxadiazolyl NH], 9.85 [s, 1H (exch.), amide NH], 7.83-7.10 (m, 20H, ArH's), 5.90 (s, 1H, pyrazolinyl $\mathrm{CH}$ ); Anal. for $\mathrm{C}_{30} \mathrm{H}_{23} \mathrm{~N}_{5} \mathrm{O}_{2} \mathrm{~S}$ Calcd.: C, 69.61; H, 4.48; N, 13.53; S,6.19. Found: C, 69.50; H, 4.50; N, $13.59 ; \mathrm{S}, 6.15$.

2-[5-Benzoylamino-1,3,-diphenyl-4-(2-methoxyphenyl)-2-pyrazolin-5-yl]-1,3,4-oxadiazole-5(4H)thione (12b). Using the general procedure, 8b gave 12b $(81 \%)$; mp. $140^{\circ} \mathrm{C}$; IR $\left(\left(\mathrm{cm}^{-1}\right): 3375,3139\right.$ (NH str.), 3067 (aromatic $\mathrm{CH}$ str.), 2938, $2836\left(\mathrm{OCH}_{3}\right.$ and pyrazolinyl aliphatic $\mathrm{CH}$ str.), 1682 (amide $\mathrm{C}=\mathrm{O}$ str.), 1602 (aromatic $\mathrm{C}=\mathrm{C}$ str.), 1498, 1470, 1446 (NH bend.), 1343, 1328, 1293, 1253 (C-N str.), 1190, 1149, 1106, 1027 (=C-O-C= str., $\mathrm{C}-\mathrm{O}$ str. of $\left.\mathrm{OCH}_{3}\right), 1069$ (C=S str.), 759, 699 (aromatic $\mathrm{CH}$ bend.); ${ }^{1} \mathrm{H}-\mathrm{NMR}$ (DMSO-d $\mathrm{d}_{6}$ ): $\delta 14.52$ [brs, $1 \mathrm{H}$ (exch.), oxadiazolyl NH], 9.69 [s, $1 \mathrm{H}$ (exch.), amide $\mathrm{NH}$, 7.78-6.78 (m,19H, ArH's), $6.32(\mathrm{~s}, 1 \mathrm{H}$, pyrazolinyl $\mathrm{CH}), 3.85\left(\mathrm{~s}, 3 \mathrm{H}, \mathrm{OCH}_{3}\right)$; Anal. for $\mathrm{C}_{31} \mathrm{H}_{25} \mathrm{~N}_{5} \mathrm{O}_{3} \mathrm{~S}$ Calcd.: C, 67.99; H, 4.60; N, 12.79; S,5.85. Found: C, 68.12 H, 4.62; N, 12.69; S,5.82.

$N$-(N-Phenylthiocarbamoyl)-5-benzoylamino-1,3-diphenyl-4-(2-methoxyphenyl)-2-pyrazoline-5-carbohydrazide (13). To a suspension of $\mathbf{8 b}(1 \mathrm{mmol})$ in absolute ethanol $(25 \mathrm{~mL})$ was added phenyl isothiocyanate $\left(1.01 \mathrm{mmol}\right.$ ), and the mixture was heated under reflux for $2 \frac{1}{2}$ hours (all materials went into solution after 45 minutes of heating under reflux). The reaction mixture was concentrated, allowed to cool, and upon scratching, the product precipitated as colorless crystals. The crude product was collected by filtration and recrystallized from ethanol as colorless crystals of $13(75 \%)$ mp.145-146 ${ }^{\circ} \mathrm{C}$; IR ( $\mathrm{cm}^{-1}$ ): 3371, 3327, 3150, 3110 (NH str.), 3059,3029 (aromatic CH str.), 2964, 2923, 2880, 2842 $\left(\mathrm{OCH}_{3}\right.$ and pyrazolinyl aliphatic $\mathrm{CH}$ str.), 1718 (hydrazide $\mathrm{C}=\mathrm{O}$ str.), 1678 (amide $\mathrm{C}=\mathrm{O}$ str.), 1651, 1625 (C=N str.), 1599 (aromatic $\mathrm{C}=\mathrm{C}$ str.), 1543,1497, 1473, 1442 ( $\mathrm{NH}$ bend.), 1380, 1357, 1320, 1291, 1252 (C-N str.), 1210-1025 (C-O str.), 1070 (C=S str.), 758, 692 (aromatic CH bend.); ${ }^{1} \mathrm{H}-\mathrm{NMR}$ (DMSO-d ${ }_{6}$ ): $\delta 10.79,9.92,9.34,9.15$ [3s, 1brs, 4H (exch.), 4NH], 7.80-6.55 (m, 24H, ArH's), 6.23 (s, $1 \mathrm{H}$, pyrazolinyl $\mathrm{CH}$ ), $3.76\left(\mathrm{~s}, 3 \mathrm{H}, \mathrm{OCH}_{3}\right)$; Anal. for $\mathrm{C}_{37} \mathrm{H}_{32} \mathrm{~N}_{6} \mathrm{O}_{3} \mathrm{~S} \mathrm{Calcd} .: \mathrm{C}, 69.35 ; \mathrm{H}, 5.03 ; \mathrm{N}, 13.11$; S, 5.04. Found: C, 69.1, H, 5.30; N, 13.00; S, 5.10

\section{References}

1. Polanc, S. Recent Application of Hydrazides and Related Compounds for The Synthesis Of Heterocycles. Targets Heterocycl. Syst. 1999, 3, 33-91 [ Chem Abstr. 2000, 133, 237877u].

2. Dutta, M. M.; Goswami, B. N.; Kataky, J. C. S. Studies on Biologically Active Heterocycles. Part I. Synthesis and Antifungal Activity of Some New Aroyl Hydrazones and 2,5-Disubstituted1,3,4-oxadiazoles. J. Heterocyclic Chem. 1986, 23, 793-795 and references cited therein.

3. Yale, H. L.; Losee, K.; Martins, J.; Holsing, M.; Perry, F. M.; Bernstein, J. Chemotherapy of Experimental Tuberculosis. VIII. The Synthesis of Acid Hydrazides, Their Derivatives and Related Compounds. J. Am. Chem. Soc. 1953, 75, 1933-1942. 
4. Bernstein, J.; Lott, W. A.; Steinberg, B. A.; Yale, H. L. Chemotherapy of Experimental Tuberculosis. V. Isonicotinic Acid Hydrazide (Nydrazid) and Related Compounds. Am. Rev. Tuberc. 1952, 65, 357-364 [Chem. Abstr. 1953, 47, 12623c].

5. Bernstein, J.; Jambor, W. P.; Lott, W. A.; Pansy, F. E.; Steinberg, B. A.; Yale, H. L. Chemotherapy of Experimental Tuberculosis. VI. Derivatives of Isoniazid. Am. Rev. Tuberc. 1953, 67, 354-365 [Chem. Abstr. 1954, 48, 3551f].

6. Bernstein, J.; Jambor, W. P.; Lott, W. A.; Pansy, F.; Steinberg, B. A.; Yale, H. L. Chemotherapy of Experimental Tuberculosis. VII. Heterocyclic Acid Hydrazides and Derivatives. Am. Rev. Tuberc. 1953, 67, 366-375 [Chem. Abstr. 1954, 48, 3551g].

7. Erman, P. H.; Straub, H. Heterocyclic Hydrazide Derivatives of Monocyclic $\gamma$-Lactam Antibiotics. U.S. Pat. US 5,318,963, Jun 7, 1994 [Chem. Abstr. 1995, 122, 55819s].

8. Wu, E. S. C.; Kover, A.; Loch, J. T., III; Rosenberg, L. P.; Semus, S. F.; Verhoest, P. R.; Gordon, J. C.; Machulskis, A. C.; Mc Creedy, S. A.; Zongrone, J. Acylhydrazones as $\mathrm{M}_{1} / \mathrm{M}_{3}$ Selective Muscarinic Agonists. Bioorg. Med. Chem. Lett. 1996, 6, 2525-2530 [Chem. Abstr. 1997, 126, 69739k].

9. Markham, P. N.; Klyachko, E. A.; Crich, D.; Jaber, M. R.; Johnson, M. E.; Mulhearn, D. C.; Neyfakh, A. A. Bactericidal Antimicrobial Methods and Compositions Using Acyl Hydrazides, Oxyamides, and 8- Hydroxyquinolines as Antibiotic Potentiators for Treatment of Gram-Postive Infections. PCT Int. Appl. WO 01 70, 213, Sep 27, 2001 [Chem. Abstr. 2001, 135, 251941h].

10. Troeberg, L.; Chen, X.; Flaherty, T. M.; Morty, R. E.; Cheng, M.; Hua, H.; Springer, C.; Mc Kerrow, J. H.; Kenyon, G. L.; Lonsdale-Eccles, J. D.; Coetzer, T. H. T.; Cohen, F. E. Chalcone, Acyl Hydrazide, and Related Amides Kill Cultured Trypanosoma Brucei Brucei. Mol. Med. (N.Y.) 2000, 6, 660-669 [Chem. Abstr. 2001, 134, 246896x].

11. Broadhurst, M. J.; Johnson, W. H.; Walter, D. S. Preparation of Hydroxycarbamoylalkylcarboxylic Acid Azacyclic Hydrazides as TNF- $\alpha$ Inhibitors. PTC Int. Appl. WO 00 35,885, Jun 22, 2000 [Chem. Abstr. 2000, 133, 58802u].

12. Milyutin, A. V.; Safonva, N. V.; Chesnokov, V. P.; Nazmetdinov, F. Y.; Voronina, E. V.; Krylora, I. V.; Andreichikov, Y. S.; Kolla, V. E.; Kozhevnikov, Y. V. Synthesis, Properties, and Biological Activity of $\beta$-Aroylpyruvylhydrazides of N-Methyl and N-Phenylanthranilic Acids. Khim.-Farm. Zh. 1996, 30, 26-28 [Chem. Abstr. 1996, 125, 185294q].

13. Silvestrini, B; Cheng, C. Y. Preparation of 3-Substituted-1-Benzyl-1H-Indoles as Antifertility Agents. U.S. Pat. US 6,001,865 Dec. 14, 1999 [Chem. Abstr. 2000, 132, 22964p].

14. Sengupta, A. K.; Bhatnagar, A. Synthesis and Antimicrobial Screening of [[1-(4Methyl/chlorophenyl)-1H-tetrazolo-5-yl]thio]acetic Acid [N-Substituted-phenyl)methylene]hydrazides]. J. Indian Chem. Soc. 1987, LXIV, 616-619.

15. Opie, T. R. Preparation of Benzodioxincarboxylic Acid Hydrazides as insecticides. Eur. Pat. Appl. EP 984,009, Mar 8, 2000 [Chem. Abstr. 2000, 132, 194382p].

16. Tomotaki, Y.; Kamiya, K.; Abe, Y. Deodorant Compositions. PTC Int. Appl. WO 01 62,309, Aug 30, 2001 [Chem. Abstr. 2001, 135, 199698t]. 
17. Eid, M. M.; Badawy, M. A.; Mansour, A. K. Reactions of 3-Hydrazino-5,6-diphenyl-1,2,4triazine with 4-Arylidene-2-phenyl-5(4H)-oxazolones and 4-Benzylidene-3-methyl-5(4H)isoxazolone. J. Heterocyclic Chem. 1998, 25, 1813-1815.

18. Eid, M. M. Reaction of 3-Hydrazino-5,6-diphenyl-1,2,4-triazine with Some Hexoses and Pentoses. J. Carbohydr. Chem. 1999, 10, 461-468.

19. Coutouli-Argyropoulou, E.; Argyropoulos, N. G.; Thessalonikeos, E. 1,3-Dipolar Cycloaddition Reactions of 2-Phenyl-4-arylideneoxazol-5(4H)-ones with Nitrile Imines. A Reinvestigation of The Regiochemistry of The 1,3- Dipolar Cycloaddition Reactions of 2-Phenyl-4-Arylideneoxazol-5(4H)-ones with Nitrile Oxides. J. Chem. Res. 1990, (S) 202-203; (M) 1557.

20. Shawali, A., S.; Fahmi, A.A.; Hassaneen, H. M.; Abdallah, M. A.; Abdelhamid, H. A. 1,3Dipolar Cycloaddition Rections of Diphenylnitrilimine with Esters of $\alpha, \beta$-Didehydro Amino Acids. J. Chem. Res. 1992, (S) 360-361; (M) 2936-2944.

Sample availability: Not available.

(C) 2003 by MDPI (http:www.mdpi.org). Reproduction is permitted for noncommercial purposes. 